\title{
Technique of tooth relieving of worm hobs for cutting Novikov gears with double lines of action
}

\author{
Alexander Sandler ${ }^{1}$ and Sergey Lagutin ${ }^{2}$ \\ ${ }^{1}$ LLC “SELLACTION”, 127540, Russia, Moscow, Dubninskaya St., 12-1-269 \\ ${ }^{2}$ JS Co "EZTM“", 144005, Russia, Electrostal
}

\begin{abstract}
Worm hobs for cutting Novikov gears have the profile of a generating worm, which varies not only in a radius, but also in a sign of curvature. A technique for relieving tooth flanks of these hobs by disk grinding wheel is developed. It allows for bringing together grinding and ground profiles in an axial section of the generating worm as close as possible. A method for calculating setting-ups and profile coordinates of the grinding wheel is given for real production conditions.
\end{abstract}

Key words: worm hobs, grinding, relieving, tooth profile, Novikov gears.

\section{Introduction}

Formation of screw and relieved surfaces by disk grinding wheels includes inevitable deviation of ground surface and wheel profiles. Such a deviation was called an organic error. For a straight basic rack the organic error has to be minimized as much as possible or the obtained profile is taken as the basic rack at the attained degree of minimization [1-3]. For the screw and relieved surfaces when a profile has a substantial and variable curvature, this organic error must be eliminated to the maximum degree.

This problem was solved in a well-known example for the unruled worm of ZT2 type, proposed by F.L. Litvin in 1961 (later, in the monograph [4] such worms are considered as worms ZF-II type). An axial profile of the grinding wheel was set in the form of a circle arc, and then an angle of installation of the grinding wheel axis and an axial profile of a worm thread were sought based on the condition that this arc was a contact line of the wheel and the ground surface. Obviously, a solution of an inverse problem is required to obtain a worm hob with relieved surfaces of teeth being close to the found worm thread profile.

In $[2,5]$, authors proposed the solution of a similar problem for gears with liquid friction with respect to the convex thread profile of a worm and a hob for cutting teeth. It was specified for the substantially curvilinear axial profile of the working worm (or the hob generating worm) and also taking into account a profile of a grinding wheel in the axial section of the worm. The setting angle of the grinding wheel axis was determined from the condition that this axis intersects two normal lines to the thread axial profile and it is located in a plane parallel to the axis of the worm. The proposed method ensures minimization of the organic error in the profiling of the worm thread. Similar organic error in tool profiling was accounted, because of the necessary identity of the profile of the hob generating worm.

In [6] authors made the first attempt to apply general principles of this method to the study of the relieving process of worm hobs for cutting Wildhaber-Novikov gears. In this case, two significant factors were taken into account. At first, a profile curvature of a generating rack of these worm hobs is a variable not only in a radius of curvature, but also in its sign. At second, the possibilities of a relieving machine allow for setting the axis of a grinding wheel in a plane not parallel to the axis of the ground product; it improves grinding conditions for a number of parameters.

In the present work, this research is developed and continued. It solved the main issues of relieving technique that are functionally oriented for solving the following problems: providing rear angles of the teeth necessary for the wear resistance of cutting edges of a hob, minimizing the organic error of a profile of a generating rack, determining the main parameters for setting up a relieving machine and profile of a grinding circle.

\section{Wildhaber-Novikov gears. Parameters of the basic rack}

For the first time, helical gears with an initial point tangency of the circular-helical surfaces of the teeth were proposed by the eminent American Inventor E. Wildhaber in 1926 in US Patent No 1,601,750. However, at that time this invention was unnoticed, and such gears received rapid development only after $\mathrm{M}$. L. Novikov had formulated a general principle of their formation and showed that such gears will have the increased load capacity, primarily in a contact endurance [7].

Initially, M. L. Novikov proposed a version of helical overcentrode gears, in which tooth profiles, convex on a

\footnotetext{
Corresponding author: lagutin@eztm.ru
} 
pinion and concave on a wheel, were delineated with circle arcs in the face section of gears. Later, V. N. Kudryavtsev showed that the same effect could be achieved in the gears synthesized based on two incongruent basic racks with the circle arc profiles and cut by two worm hobs: one for the pinion with convex teeth, the other for the wheel with concave ones [8].

However, at present, basic racks are standardized and widely used in production with a full tooth profile, which provide in gears two lines of action and allow for cutting the pinion and the wheel by one worm hob [9]. In particular, according to the Russian Standard GOST 15023-76, the basic rack consists of a convex addendum, a concave dedendum and a short straight section between them. Geometry of Novikov gears with two lines of action is calculated acc. Russian Standard GOST 1774472 .

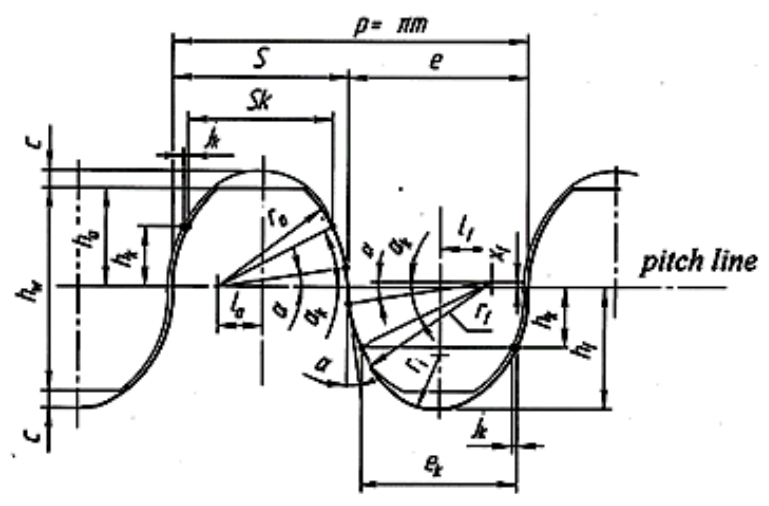

Fig. 1. Basic rack for Novikov gears acc. GOST 15023-76.

Fig. 1 shows the main parameters of the basic rack, the values of which depend on the range of modules. The pressure angle $\alpha_{\mathrm{k}}$ at the predetermined contact points on the tooth addendum and dedendum is equal $27^{\circ}$ for any case. These points are also characterized by parameters: distance $h_{\mathrm{k}}$ from the pitch line, hob tooth width $e_{\mathrm{k}}$ at its addendum and space width $S_{\mathrm{k}}$ at its dedendum, the difference $j_{\mathrm{k}}=e_{\mathrm{k}}-S_{\mathrm{k}}$ provides a backlash between the teeth of the wheels to be cut. The pressure angle $\alpha$ in the straight section of the tooth depends on the module and is equal to $\approx 8^{\circ}$.

The hob tooth in the normal section is formed according to the dimensions of the space of the basic rack. Similarly, the space between the hob teeth forms the tooth of the wheel to be cut.

The convex profile of hob tooth is outlined by a radius $r_{f}$, the center of curvature of which is located at a distance $l_{\mathrm{f}}$ from the axis of symmetry of the head and is shifted relative to the pitch line to the hob axis by $x_{\mathrm{f}}$. The center of the concave arc radius $r_{\mathrm{a}}$ of the hob tooth space is located on the pitch line, at a distance $l_{\mathrm{a}}$ from the axis of symmetry of the space. Dimensions along the pitch line are: the thickness $e$ of the hob tooth and the width $S$ of the hob space. The height $h_{\mathrm{f}}$ of the hob tooth head is equal to the dedendum of the gear to be cut. The depth of the space is equal to the sum of the addendum $h_{\mathrm{a}}$ of the gear and the radial clearance $c$ between the gear and the hob when cutting. Also in Fig. 1 the height $h_{\mathrm{w}}$ of the tooth active profile in the gear and the radii $r_{\mathrm{i}}$ of the arc transition curves on the head and in the space of the hob tooth are indicated.

\section{Worm hob parameters}

The main parameters of any worm hob are: axial module $m$, radius $r_{\mathrm{F}}$ of the pitch cylinder, number of threads $z_{0}$, screw parameter $p=0,5 m z_{0}$ of the generating surface, number of teeth $z_{\mathrm{f}}$ in the face section. From this data the lead angle $\gamma_{1}$ of the helical surface of the cutting edges on the hob pitch cylinder is determined from the expression:

$$
\tan \gamma_{1}=p / r_{\mathrm{F}}
$$

The worm hob design profile (a generating rack) is considered in the normal section of the generating worm, which is tangent to the screw front surface. For singlethread hobs this section and front surface practically coincides in the profiling zone.

When teeth are cut on the gear hobbing machine, the plane of the generating rack is installed perpendicular to the direction of the teeth of the cut wheel, while the hob axis is set taking into account the lead angle of the hob front surface.

When the teeth of the worm hob are relieved, its axis is installed in the centers of the grinding-relieving machine, that is, in the horizontal plane. And for a reliable reproduction of the profile of the generating rack in the normal section of the hob, with the adopted profiling method it is necessary to recalculate the parameters of the profile of the generating rack into the axial section of the tooth surface to be relieved.

First of all, it is necessary to determine the axial profile of the helical surface, on which the cutting edges of the hob teeth are located. To do this the prescribed angle $\alpha$ of the normal profile of the straight section should be replaced by an angle $\alpha_{1}$ of the axial profile according to the formula:

$$
\tan \alpha_{1}=\tan \alpha / \cos \gamma_{1}
$$

The curvature radii $\rho_{\mathrm{a}}$ and $\rho_{\mathrm{f}}$ of the two parts of active profile in the normal section are replaced by the corresponding radii $r_{\mathrm{a} 0}$ and $r_{\mathrm{f} 0}$ in the axial section. For single-thread hobs, these radii with a sufficient approximation are determined by the Meunier theorem from the expressions:

$$
r_{\mathrm{a} 0, \mathrm{f} 0}=\rho_{\mathrm{a}, \mathrm{f}} / \cos \gamma_{1}
$$

The arc radius $\rho_{\mathrm{i}}$ on the head of the hob tooth, which is processed fillet of the wheel tooth, in the normal section is replaced with the corresponding radius $r_{0}$ in axial section of the hob. According to Euler's formula, these radii are related by the expression:

$$
r_{0}=\rho_{\mathrm{i}} / \cos ^{2} \gamma_{1}
$$

The thread thickness $b$ in the axial section should be determined on the minimum ground radius $r_{\min }$ of the 
hob, proceeding from the thickness $b_{\mathrm{n}}$ of the normal section at the end point of machining:

$$
b=b_{\mathrm{n}} / \cos \gamma_{(\mathrm{r} \min )}
$$

where $\gamma_{(\mathrm{r} \min )}=\operatorname{atan}\left(p / r_{\min }\right)$ is the lead angle on the hob dedendum cylinder.

The calculated coordinates of the axial profile of the helical surface of the cutting edges are related to the coordinates of the generating rack profile by the dependencies:

- for point $A_{1}$ on the tooth dedendum: $x_{01}=x_{\mathrm{n} 1}$;

$z_{01}=z_{\mathrm{n} 1} / \cos \gamma_{\mathrm{x} 01} ; \tan \alpha_{01}=\tan \alpha_{\mathrm{n} 1} / \cos \gamma_{\mathrm{x} 01}$,

where $z_{\mathrm{n} 1}=0,5 \pi m_{\mathrm{n}}+l_{\mathrm{a}}-\left(r_{\mathrm{F}}-x_{\mathrm{n} 1}\right) \cot \alpha_{\mathrm{n} 1} ; \gamma_{\mathrm{x} 01}=\operatorname{atan}\left(p / x_{01}\right)$,

- for point $A_{2}$ on the tooth addendum: $x_{02}=x_{\mathrm{n} 2} ; z_{02}=$ $z_{\mathrm{n} 2} / \cos \gamma_{\mathrm{x} 02} ; \tan \alpha_{02}=\tan \alpha_{\mathrm{n} 2} / \cos \gamma_{\mathrm{x} 02}$,

where: $z_{\mathrm{n} 2}=\left(x_{\mathrm{n} 2}-r_{\mathrm{F}}+x_{\mathrm{f}}\right) \cot \alpha_{\mathrm{n} 2}-l_{\mathrm{f}} ; \gamma_{\mathrm{x} 02}=\operatorname{atan}\left(p / x_{02}\right)$.

The found parameters of the axial section of the helical surface of the cutting edges of the hob teeth are also parameters of the axial section of the relieved surfaces of the teeth.

For clarity, let us consider Figure 2, which shows an example of the basic rack for gears with the module $m_{\mathrm{n}}=$ $12 \mathrm{~mm}$.

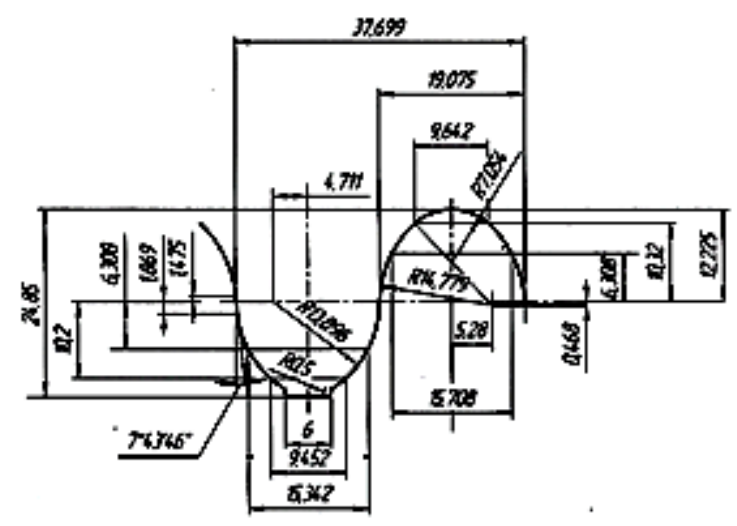

Fig. 2. An example of the basic rack of the hob for gears with the module $m_{\mathrm{n}}=12 \mathrm{~mm}$.

For the full-profile Novikov gears, the pitch line of basic rack divides the tooth height by half. The active parts of the hob tooth profile are limited by the dimensions 10.2 and $10.32 \mathrm{~mm}$ from the pitch line. At the same points, there is a conjunction of the radius sections of the profile. The tooth thickness in the normal section at the minimum machining diameter is $b_{\mathrm{n}}=$ $31.7 \mathrm{~mm}$.

The predesigned parameters of the worm cutter are: the outer diameter of $180 \mathrm{~mm}$, the number of hob threads $z_{0}=1$, the number of teeth in the face section $z_{\mathrm{f}}=$ 10 , the pitch diameter of 155.55 or its radius $r_{\mathrm{F}}=77.775$ $\mathrm{mm}$, the fall of the relieving cam on the outer cylinder $K$ $=10 \mathrm{~mm}$.

The calculated parameters are: the axial module $m$ $=12.038$; the screw parameter of the generating worm $p$ $=6.019$; the lead angle on the pitch cylinder $\gamma_{\mathrm{F}}=4.425^{\circ}$; the parameter of relieving $k=K z_{\mathrm{f}} / 2 \pi=15.915$; the minimum radius of grinding on the cylinder of hob spaces $r_{\min }=66.215$; the lead angle on this cylinder 5.194\%; the maximum tooth thickness $b=31,83$.

The parameters of the axial profile of the hob generating surface are: the minimum pressure angle on the straight section $\alpha_{1 \min }=7.7523^{\circ}$; the concave arc radius on the tooth head $r_{0 \mathrm{f}}=14.823$; the convex arc radius in the space $r_{0 \mathrm{a}}=13.938$; the radii of transitive curves on tooth head $r_{\mathrm{if}}=7.096$ and in the space $r_{\mathrm{ia}}=$ 7.002. The pressure angles at special points of the work area are:

- at a point of conjugation of the radii $r_{0 \mathrm{f}}$ and $r_{\text {if }}$ on the head of tooth $\alpha_{0 \mathrm{f}}=46.95^{\circ}$;

- at the contact point on the head of tooth $\alpha_{01}=27.35^{\circ}$;

- at a point of conjugation of the radii $r_{0 \mathrm{a}}$ and $r_{\mathrm{ia}}$ in the space $\alpha_{0 \mathrm{a}}=47.34^{\circ}$;

- at the contact point in the space $\alpha_{02}=27.08^{\circ}$.

\section{Parameters of radial-axial relieving}

The hobs for cutting involute gears have an almost straight tooth profile with the pressure angle $\alpha_{0} \approx 20^{\circ}$. The relieving of such hobs is usually carried out by the radial method.

The tooth of hobs for cutting Novikov gears has a substantially curved profile with a large difference in pressure angles. The pressure angle in the straight section of the tooth profile near the line is $\alpha_{0} \approx 8^{\circ}$, and therefore the rear angles in this section are minimal, which during cutting significantly reduces the wear resistance of the hobs, since it is precisely in this section that they are intensively formed wear sites [10]. In addition to arc sections with large pressure angles, a large shift of the relief surface profile relative to the pitch line will occur, which will appear at re-sharpening of the hob. So such hobs cannot be relieved with a purely radial method and radial-axial relieving must be used.

In general case of radial-axial relieving of the teeth of the worm hob $[1,2]$ the direction of relieving motion makes up the angle $\varphi_{c}$ with the perpendicular to the hob axis, which ensures the obtaining of a necessary rear angle $\lambda_{\mathrm{r}}$ near the cutting edges of the hob teeth.

The grinding wheel axis $\mathrm{O}_{\mathrm{w}}-\mathrm{O}_{\mathrm{w}}$ is turned (in the projection to the horizontal plane) at an angle $\varphi_{0}$ to the hob axis $\mathrm{O}_{1}-\mathrm{O}_{1}$ (see below Fig.5) and is simultaneously inclined to this plane by the angle $\beta_{\mathrm{w}}$. Such a method of setting the axis of the grinding wheel eliminates large differences in curvature of the grinding surface of the wheel, improves the grinding conditions and increases the life of the grinding wheel.

During the relieving, the hob rotates around its axis $\mathrm{O}_{1}-\mathrm{O}_{1}$ with the angular velocity $\omega_{1}$, the grinding wheel moves along the hob axis at a speed $p \omega_{1}$ and performs reciprocating motion with a speed $k \omega_{1}$, where $k=K z_{\mathrm{f}} / 2 \pi$ is the relieving parameter; $K$ is the recession of the Archimedean spiral of the cam of the relieving mechanism on the angular pitch of the hob teeth. The relieving carriage slides are turned relative to the perpendicular to the hob axis by an angle $\varphi_{c}$ which allows increasing the rear angle $\lambda_{\mathrm{r}}$ on the lateral surface of the tooth. 
The current radius $r_{\mathrm{w}}$ of the grinding wheel is determined at the point of its contact with the tooth of the hob on its current cylinder. For the initial position of the wheel, it is advisable to take the tangency of its maximum radius $r_{\mathrm{wm}}$ with the minimum radius of the work piece surface $r_{\mathrm{i} \text { min }}$.

With radial-axial relieving, the lead angle $\gamma_{0}$ of the relieved surface at each of two selected points $\left(i_{1,2}\right)$ is determined from the expression $[1,2]$

$$
\tan \gamma_{0 \mathrm{i}}=\left[p \pm k \sin \left(\alpha_{\mathrm{i}}+\varphi_{\mathrm{c}}\right) / \cos \alpha_{\mathrm{i}}\right] / x_{0 \mathrm{i}}
$$

where the radius $x_{0 \mathrm{i}}$ of the location of the selected point on the ground surface is determined from the drawing of the product.

The sign "+" in square brackets refers to the teeth side where the lead angle of the relieved surface is greater than the lead angle of the generating surface of the hob, sign "minus", respectively, to the opposite side of the teeth.

From formula (6) it follows that the values of the angles $\gamma_{0 \mathrm{i}}$ depend on the angle $\varphi_{\mathrm{c}}$ of the installation of the relieving support, which is determined by the required rear angle near the cutting edge of the tooth. For hobs with variable profile curvature, this angle is determined at the location of smallest value of angle $\alpha_{0 \mathrm{i}}$ and equal to $\alpha_{0 \min }=7.7523^{\circ}$ in the considered example for a straight line segment near the pitch line.

\section{Calculation of the angle $\varphi_{c}$ installation of the relieving support of the machine}

For worm hobs intended for finish cutting of involute gears with module $12 \mathrm{~mm}$ of the thermally hardened steels, the Russian State Standard 9324-80 recommends radial relieving parameters providing the normal relief angle on a pitch cylinder $\approx 4^{\circ}$. Based on this recommendation, for the considered type of hobs, we establish the following dependence, which ensures the determination of $\varphi_{c}$ with the sufficient accuracy for practical calculations:

$$
\left(\tan \gamma_{0 \mathrm{~F}}-\tan \gamma_{\mathrm{xF}}\right) \cos \alpha_{0 \min }=\tan 4^{\circ}
$$

Expanding the meanings of $\tan \gamma_{0 \mathrm{~F}}$ from (6) and tan $\gamma_{\mathrm{xF}}=p / r_{\mathrm{F}}$, we obtain the following expression for determining the value of $\varphi_{\mathrm{c}}$

$$
\sin \left(\alpha_{0 \min }+\varphi_{\mathrm{cR}, \mathrm{L}}\right)=0,44 r_{\mathrm{F}} /\left(K z_{\mathrm{f}}\right)
$$

Indexes $\mathrm{R}$, $\mathrm{L}$ relate to the right and left sides of the hob tooth. For the example considered above with $\alpha_{0 \min }=7.7523^{\circ}$ we obtain $\varphi_{\mathrm{cR}, \mathrm{L}}=12.229^{\circ}$. Taking into account the price of rotation angle scale we set according to the rounding rules $\varphi_{\mathrm{cR}, \mathrm{L}}=12^{\circ}$.

Table 1 shows the calculated parameters of the profile of the hob tooth and the lead angles of the relieved surface of the teeth at seven key points of the profile $i=1 \ldots 7$ :

1 - the boundary of the active section of the profile on the tooth leg,
2 - the contact point on the dedendum of the hob tooth,

3 - the conjugation of the arc concave section with a rectilinear section,

4 - the point on the hob pitch cylinder,

5 - the conjugation of the rectilinear and arc sections on the tooth head,

6 - the contact point on the addendum of the hob tooth,

7 - the boundary of the active section of the profile on the tooth head.

\begin{tabular}{|c|c|c|c|c|c|}
\hline \multirow{2}{*}{$\begin{array}{l}\text { № } \\
\mathrm{p} / \mathrm{p}\end{array}$} & \multirow{2}{*}{$\begin{array}{l}\text { Radius } \\
\text { of } \\
\text { point, } \\
\text { mm } \\
x_{\mathrm{i}}=r_{\mathrm{i}} \\
\end{array}$} & \multirow{2}{*}{$\begin{array}{l}\text { Pressure } \\
\text { angle in } \\
\text { axial } \\
\text { section, } \\
\alpha_{0 i}^{\circ}\end{array}$} & \multirow{2}{*}{$\begin{array}{l}\text { Lead angle } \\
\text { of screw } \\
\text { surface of } \\
\text { cutting } \\
\text { edges, } \gamma_{i}^{\circ}\end{array}$} & \multicolumn{2}{|c|}{$\begin{array}{l}\text { Lead angles of } \\
\text { relieved surface on } \\
\text { the tooth sides: }\end{array}$} \\
\hline & & & & $\begin{array}{l}\text { right, } \\
\gamma_{0 i \mathrm{R}}{ }^{\circ}\end{array}$ & $\begin{array}{l}\text { left, } \\
\gamma_{0 \text { iL }}{ }^{\circ}\end{array}$ \\
\hline 1 & 67.575 & 47.338 & 5.09 & 21.207 & -11.853 \\
\hline 2 & 71.467 & 27.079 & 4.814 & 13.598 & -4.200 \\
\hline 3 & 75.906 & 7.752 & 4.587 & 8.576 & 0.446 \\
\hline 4 & 77.775 & 7.752 & 4.425 & 8.373 & 0.435 \\
\hline 5 & 79.25 & 7.752 & 4.343 & 8.219 & 0.427 \\
\hline 6 & 84.083 & 27.35 & 4.094 & 11.679 & -3.635 \\
\hline 7 & 88.095 & 46.951 & 3.909 & 16.439 & -9.001 \\
\hline
\end{tabular}

Table 1. Parameters of hob tooth profile in key points (for the given example).

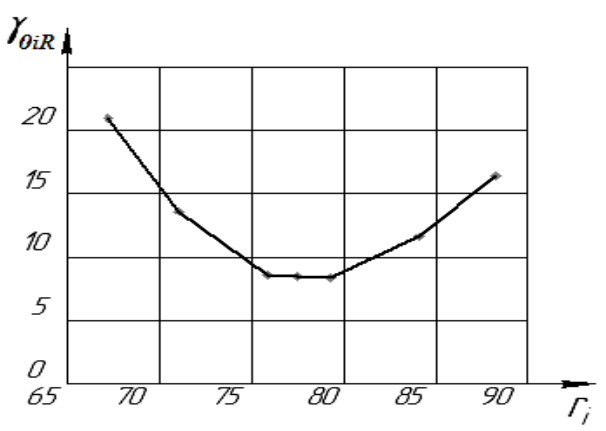

Fig. 3. Diagram of the function $\gamma_{0 i R}=f\left(r_{i}\right)$ - right flank.

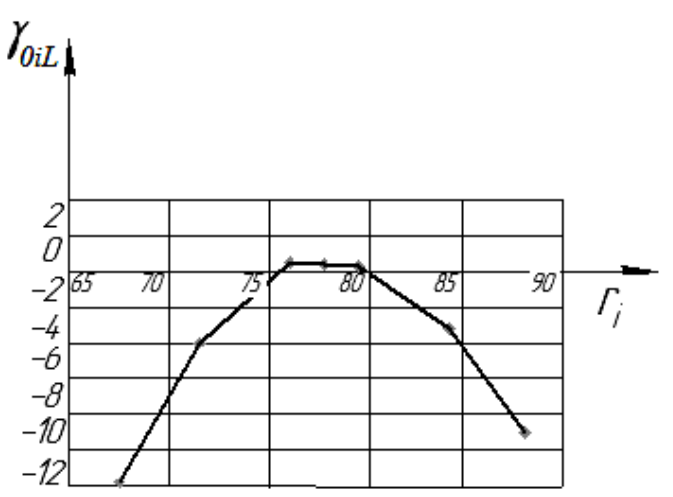

Fig. 4. Diagram of the function $\gamma_{0 \mathrm{iL}}=f\left(r_{\mathrm{i}}\right)$ - left flank.

In Fig. 3 and 4 the graphs of the dependence of the lead angles of the relieved surface on the radius $x_{0 \mathrm{i}}=r_{\mathrm{i}}$ 
of the profile point, taking into account the pressure angle $\alpha_{0 \mathrm{i}}$ at $\varphi_{\mathrm{c}}=12^{\circ}$ are shown for both sides of tooth.

The analysis of the calculated data shows that the lateral surfaces of teeth of a hob are organically characterized by the presence of paired points with equal lead angles of the relieved surface, but the values of these angles differ significantly from the lead angle of the helical generating surface.

\section{Choice of the designed points to construct designed normals}

The choice of two points of the axial profile of the relieved surface with the parameters: $A_{1}\left(x_{01}, z_{01}, \alpha_{01}\right)$ and $A_{2}\left(x_{02}, z_{02}, \alpha_{02}\right)$, must satisfy two conditions. Firstly, the part between the selected points should cover the active part of the tooth profile as much as possible, including contact points $\left(\alpha_{0} \approx 27^{\circ}\right)$. Secondly, undercutting of the tooth cutting edges should be excluded.

To do this, on the right side of the teeth of the righthand hob the angle $\beta_{\mathrm{w}}$ of setting the axis of the grinding wheel in a plane parallel to the hob axis should not exceed significantly the maximum lead angle of the generating surface. When relieving the opposite side of the tooth, the angle $\beta_{\mathrm{w}}$ should not be substantially less than the lead angle of the helical surface of the cutting edges on the tooth head.

Point $A_{1}$ should be selected on the tooth leg of the hob. A study conducted with specific examples (see the calculation example below) showed that these conditions are most fully satisfied if, as point $A_{1}$, the design contact point in the tooth space on a concave arc at $\alpha_{01} \approx 27^{\circ}$ (point 2 in the table 1). As point $A_{2}$, we should choose the conjugation of the convex arc section with the transition curve on the tooth head at $\alpha_{02} \approx 47^{\circ}$ (point 7 in table 1). In this case, the angle $\beta_{\mathrm{w}}$ of the inclination of the wheel axis is less than the smaller of the values of the angle $\gamma_{0 \mathrm{i}}$ at the selected design points.

\section{Determination of the angle $\beta_{w}$ of inclination of the grinding wheel axis}

The design scheme for determining the angle $\beta_{\mathrm{w}}$ of the installation of the axis of the grinding wheel for radialaxial relieving of surfaces with a variable curvature profile is shown on Fig. 5.

In the coordinate system $\mathrm{XYZ}$ (Fig. 5), the $\mathrm{Z}$ axis is directed along the axis of the hob, the $\mathrm{X}$ axis is aligned with the axis of symmetry of the thread, $\mathrm{XZ}$ is the plane of the axial section of the hob, and the $\mathrm{Y}$ axis is perpendicular to this section. The plane of the axis of the grinding wheel is parallel to the $\mathrm{Y}$ axis, intersects the $\mathrm{X}$ axis at a distance $a$ from the $Z$ axis under an angle $\varphi_{0} \neq 0$.

As shown in the lower projection of Fig. 5, the value of distance $a$ is determined as:

$$
a=r_{\mathrm{imin}}+0.5 b \tan \varphi_{0}+r_{\text {ит }} / \cos \varphi_{0}
$$

where $b$ is the thickness of the worm thread being ground with a minimum radius $r_{\mathrm{i} \text { min }}$.

The equation of the plane containing the axis of the grinding wheel is written in the form:

$$
x(z)=a-z \tan \varphi_{0}
$$

Coordinates $z_{\mathrm{i}}$ of the intersection of normals recovered from points $A_{1}$ and $A_{2}$ with the plane of the axis of the grinding wheel:

$$
z_{\mathrm{i}(1,2)}=\left(a-x_{0 \mathrm{i}}+z_{0 \mathrm{i}} \tan \alpha_{\mathrm{i}}\right) /\left(\tan \alpha_{\mathrm{i}}+\tan \varphi_{0}\right)
$$

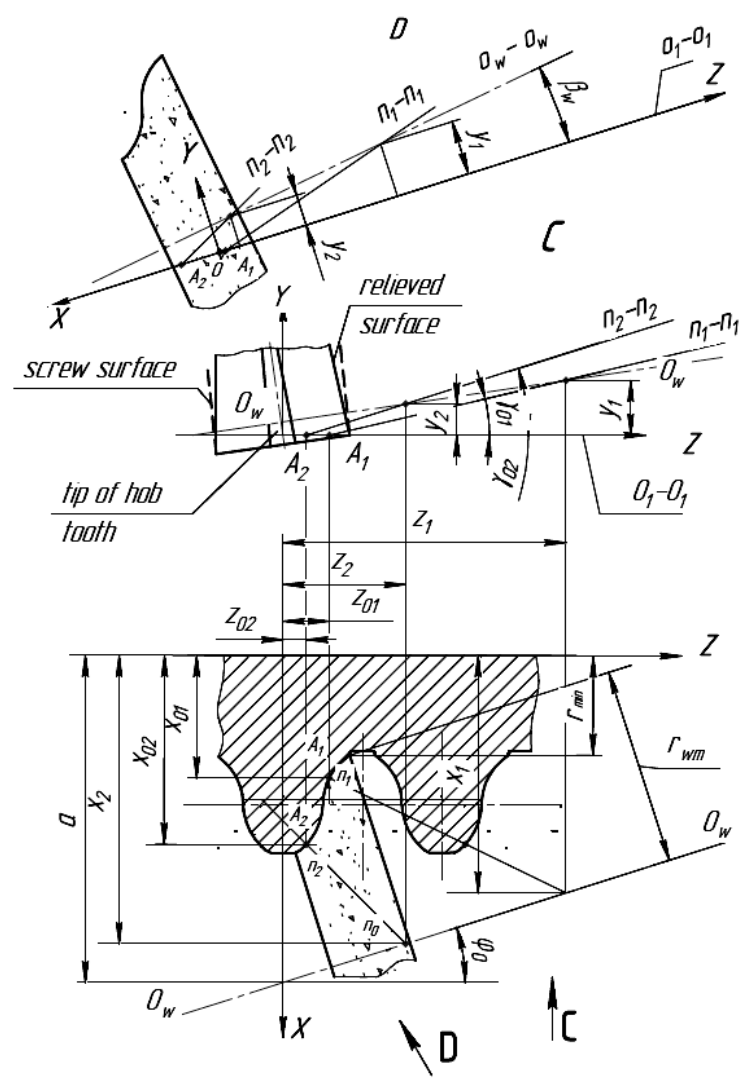

Fig. 5. The design scheme of the installation of the axis of the grinding wheel.

The two other coordinates $x_{\mathrm{i}(1.2)}$ and $y_{\mathrm{i}(1.2)}$ of each of the intersection points of the normals with this plane are determined through $z_{\mathrm{i}}$. When determining the coordinate $y_{\mathrm{i}(1.2)}$ we should consider the frontal projection (view C):

$$
x_{\mathrm{i}(1.2)}=a-z_{\mathrm{i}} \tan \varphi_{0} ; y_{\mathrm{i}(1.2)}=\left(z_{\mathrm{i}}-z_{\mathrm{oi}}\right) \tan \gamma_{0 \mathrm{i}}
$$

The angle $\beta_{\mathrm{w}}$ of the inclination of the axis of the grinding wheel is determined in the tangent function (from top view on the plane of the axis of the grinding wheel) by the formula:

$$
\tan \beta_{\mathrm{w}}=\frac{y_{1}-y_{2}}{\sqrt{\left(x_{1}-x_{2}\right)^{2}+\left(z_{1}-z_{2}\right)^{2}}}
$$

\section{Choice of angle $\varphi_{0}$ for relieving the tooth flanks}

As mentioned above, turning the hob axis by the angle $\varphi_{0}$, improves grinding conditions by increasing the 
curvature of the surface of the wheel at the points of contact with a processed surface, and reduces the difference between the angle $\beta_{\mathrm{w}}$ of the inclination of the wheel axis and the lead angles of the helical line of the cutting edges of the hob teeth. The choice of the angle $\varphi_{0}$ is determined by two restrictions. On the one hand, it is necessary to minimize the mentioned difference of angles $\beta_{\mathrm{w}}$ and $\gamma_{0 \mathrm{i}}$. On the other hand, the value $\varphi_{0}$ should not lead to pruning of the cutting edges of the teeth on the opposite side of the thread of the generating surface, i.e., the grinding wheel should enter the space without damaging the adjacent tooth.

Consider the issue of pruning prevention in more detail. The minimum distance between the back (flat) side of the grinding wheel and the opposite side of the thread of the generating surface takes place opposite to the most protruding point on the head of the hob tooth. Such a point is a contact point on the tooth head with a pressure angle $\alpha_{0 \mathrm{i}} \approx 27^{\circ}$.

Due to the difference between the angle $\beta_{\mathrm{w}}$ of the wheel inclination and the lead angle $\gamma_{i}$ of the generating surface, the dangerous approach of the surfaces is shifted from the horizontal plane (downwards when processing the right side of the tooth). Its value reaches maximum at the extreme point of intersection of the face sections of the outer wheel cylinder (radius $r_{\mathrm{wm}}$ ) and the cylinder of the hob radius $\left(r_{\mathrm{F}}+h_{\mathrm{k}}\right)$ at the contact point on the tooth head. The distance from this point to the horizontal plane for simple geometric reasons is determined by the formula:

$$
l_{\max }=r_{\mathrm{wm}} \sin \psi,
$$

where

$$
\psi=\operatorname{acos} \frac{r_{\mathrm{wm}}^{2}+\left(r_{\mathrm{wm}}+r_{\mathrm{min}}\right)^{2}-\left(r_{\mathrm{F}}+h_{\mathrm{k}}\right)^{2}}{2 r_{\mathrm{wm}}\left(r_{\mathrm{wm}}+r_{\min }\right)}
$$

The linear displacement $\Delta$ of the back surface of the grinding wheel to the middle of the space at a length $l_{\max }$ in a screw projection onto the axial plane of the cutter to both tooth sides will be:

$$
\Delta_{\mathrm{R}, \mathrm{L}} \approx \pm l_{\max }\left[\tan \beta_{\mathrm{wR}, \mathrm{L}} \cos \varphi_{0}-p /\left(r_{\mathrm{F}}+h_{\mathrm{k}}\right)\right]
$$

where the sign "+" relates to right side of the right-hand hob, the sign "_." to its left side.

In the angular dimension, this displacement is determined from the expression:

$$
\tan \varphi_{\beta}=\Delta /\left(r_{\mathrm{F}}+h_{\mathrm{k}}\right)
$$

The pruning of the cutting edges of the opposite side of the thread is guaranteed to be excluded if condition $\varphi_{0}+\varphi_{\beta} \leq \alpha_{0 \mathrm{i}}$ is met, where the value $\alpha_{0 \mathrm{i}}$ is accepted for the key point 6 from Table 1. For preliminary calculation of the relieving parameters, the angle $\varphi_{0}$ should be taken equal to $\varphi_{0}=18^{\circ}$. Then, having determined the angle $\varphi_{\beta}$ by formula (19), it is possible to correct the angle $\varphi_{0}$ by increasing it to the maximum permissible value

$$
\varphi_{0 \max }=\alpha_{0 \mathrm{i}}-\varphi_{\beta}
$$

Given that increasing $\varphi_{0}$ reduces the needed value of the angle $\beta_{\mathrm{w}}$, such an adjustment is advisable.
As an example, we will calculate the relieving parameters $\beta_{\mathrm{w}}$ and $\varphi_{0}$ as applied to the hob considered above, and study the features of the resulting side surfaces.

As the initial points for constructing the designed normals, we take the points: on the leg $A_{1}$, at the contact point with pressure angle $\alpha_{01} \approx 27^{\circ}$ (key point 2 from table1), on head $A_{2}$ at the conjugation between the active profile and the transition curve with the angle $\alpha_{02} \approx 47^{\circ}$ (key point 7). The coordinates of these points and the exact values of the pressure angles are in table 1.

The maximum radius of the grinding wheel is assumed to be equal to $r_{\mathrm{wm}}=60 \mathrm{~mm}$, the minimum radius of the relieved surface on the space $r_{\min }=66.215 \mathrm{~mm}$, the height of the grinding profile of the hob tooth is $H_{1}=23.785 \mathrm{~mm}$ for both sides of the teeth of the manufacturing surface. The value of the turning angle of the relieving support to provide a rear angle in the middle section of the profile $\varphi_{\mathrm{c}}=12^{\circ}$ for both sides of the teeth.

The lead angles $\gamma_{0 \mathrm{i}}$ of the relieved surface at the points $A_{1}$ and $A_{2}$ were shown in table 1 for each side of a tooth of the right-hand hob. On the right side of the tooth, at which the lead angle of the relieved surface is greater than the lead angle of the generating worm they are equal $\gamma_{01}=13.598^{\circ}, \gamma_{02}=6.439^{\circ}$. On the opposite, for the left tooth side we have $\gamma_{01}=-4.200^{\circ}, \gamma_{02}=-9.001^{\circ}$.

Negative values of lead angles $\gamma_{0 \mathrm{i}}$ of the relieved surface at the points of arc sections of the profile mean its transformation in these sections into an analogue of the left helical surface, which is characteristic for singlethread hobs with a standard lateral rear angle of $4^{\circ}$ or more, with the number of modules in the pitch diameter of 12 or more.

\begin{tabular}{|c|c|c|c|c|}
\hline \multirow{3}{*}{ Data } & \multirow{2}{*}{$\begin{array}{c}\text { Formula } \\
\text { number }\end{array}$} & \multicolumn{3}{|c|}{ Estimated Values } \\
\hline & & \multicolumn{2}{|c|}{ preliminary } & final \\
\hline & & Right & \multicolumn{2}{|c|}{ Left tooth side } \\
\hline$\varphi_{0}^{\circ}$ & & 18.0 & 18.0 & 19.0 \\
\hline$a$, & (9) & 134.47 & 134.47 & 135.15 \\
\hline$x_{1}$ & $(12)$ & 107.76 & 107.76 & 107.21 \\
\hline$z_{1}$ & $(11)$ & 82.21 & 82.21 & 81.14 \\
\hline$y_{1}$ & (12) & 17.17 & -5.17 & -5.13 \\
\hline$x_{2}$ & $(12)$ & 122.47 & 122.47 & 122.44 \\
\hline$z_{2}$ & (11) & 36.94 & 36.94 & 36.91 \\
\hline$y_{2}$ & $(12)$ & 9.47 & -5.09 & -5.08 \\
\hline$\beta_{\mathrm{w}}^{\circ}$ & $(13)$ & 9.19 & -0.095 & -0.064 \\
\hline$\psi^{\circ}$ & $(15)$ & 34.64 & 34.64 & 34.64 \\
\hline$l_{\max }$ & $(14)$ & 34.11 & 34.11 & 34.11 \\
\hline$\Delta$ & $(16)$ & 2.81 & 2.49 & 2.47 \\
\hline$\varphi_{\beta}{ }^{\circ}$ & $(17)$ & 8.93 & 7.95 & 7.89 \\
\hline$\varphi_{0 \max }{ }^{\circ}$ & (18) & 18.42 & 19.40 & 19.46 \\
\hline
\end{tabular}

Table 2 summarizes the main stages of the calculations by means of the corresponding formulas.

Table 2. Calculation of the parameters $\beta_{w}$ and $\varphi_{0}$ of the hob teeth. 
For the right side, the difference between the values of the initial $\varphi_{0}$ and the adjusted $\varphi_{0 \max }$ angles in the preliminary calculation is less than $1^{\circ}$, therefore, a change in $\varphi_{0}$ is not required. For the left side, the final calculation is carried out by increasing the angle $\varphi_{0}$ to $19^{\circ}$, and recalculation of $\beta_{\mathrm{w}}$, which however has changed very slightly and which in practice in both cases is rounded to the value $-0.1^{\circ}$.

In $[1,2]$ it was shown that the discrepancy between the profiles of the grinding wheel surface and the hob tooth flank to be ground will be minimal if in the vicinity of the calculated contact point, the line of their contact will not be crossing the axial section of the hob, but touching it. This condition is satisfied in the case when the axis of the grinding wheel is inclined to the axial plane at an angle $\beta_{\mathrm{w}}$, which is determined depending on the pressure angle $\alpha_{0}$, the lead angle $\gamma_{0 \mathrm{i}}$ and the radius $r_{\mathrm{i}}$ of the relieved surface, taking into account the installation angle $\varphi_{0}$ of the wheel axis in the projection onto the horizontal plane, by the expression:

$$
\tan \beta_{\mathrm{w}}=\tan \gamma_{0 \mathrm{i}} \cos \alpha_{0} / \cos \left(\alpha_{0}+\varphi_{0}\right)
$$

Table 3 shows the values of the angle $\beta_{\mathrm{w}}$ calculated according to this dependence for seven key points of the axial section of the right and left sides of the hob teeth.

Table 3. Values of the angle $\beta \mathrm{w}$ calculated for the different sides of the hob tooth.

\begin{tabular}{|c|c|c|c|c|}
\hline Point numbers & 1 & 2 & 3 & 4 \\
\hline $\mathrm{x}_{0 i}, \mathrm{~mm}$ & 67.575 & 71.467 & 75.906 & 77.775 \\
\hline$\alpha_{00}{ }^{\circ}$ & 47.34 & 27.08 & 7.75 & 7.75 \\
\hline$\gamma_{0 i}{ }^{\circ}$ (right side) & 21.207 & 13.598 & 8.576 & 8.373 \\
\hline$\beta_{\mathrm{w}}{ }^{\circ}$ (right side) & 32.22 & 16.96 & 9.42 & 9.198 \\
\hline$\gamma_{0{ }^{\circ}}{ }^{\circ}$ (left side) & -11.853 & -4.20 & 0.446 & 0.435 \\
\hline$\beta_{\mathrm{w}}{ }^{\circ}$ (left side) & -18.882 & -5.29 & 0.491 & 0.479 \\
\hline Point numbers & 5 & 6 & 7 & \\
\hline $\mathrm{x}_{0 i}, \mathrm{~mm}$ & 79.25 & 84.083 & 88.095 & \\
\hline$\alpha_{0 \mathrm{i}}{ }^{\circ}$ & 7.75 & 27.35 & 46.95 & \\
\hline$\gamma_{0 \mathrm{i}}{ }^{\circ}$ (right side) & 8.219 & 11.679 & 16.439 & \\
\hline$\beta_{\mathrm{w}}{ }^{\circ}$ (right side) & 9.029 & 16.641 & 25.441 & \\
\hline$\gamma_{0{ }^{\circ}}{ }^{\circ}$ (left side) & 0.427 & -3.625 & -9.001 & \\
\hline$\beta_{\mathrm{w}}{ }^{\circ}$ (left side) & 0.470 & -4.590 & -14.325 & \\
\hline
\end{tabular}

In Fig. 6 (upper part) a graphical interpretation of the dependence (19) of the angle $\beta_{\mathrm{w}}$ on the hob radius is given for the right side of the teeth. Let us draw two boundary straight lines: one through the starting points $A_{1}$ and $A_{2}$ of the designed normals, and the second horizontal line at minimum value $\beta_{\mathrm{w}}=9.19^{\circ}$ (from Table 3 ) until they mutually intersect in some point $C$. The sections of the profile that fell into the sector between the boundary lines have practically no organic error, since any straight line drawn through the intersection point $\mathrm{C}$ inside the sector also pass through two points of the axial profile of the relieved surface. Outside the obtained sector, the relieved profile has a small organic deviation from the given profile of the grinding wheel.

At the lower part of Fig. 6 a similar dependence of the angle $\beta_{\mathrm{w}}$ on the hob radius is shown for the left side of the teeth. One boundary straight line passes through the points of the line corresponding to the values of the radius at points $A_{1}$ and $A_{2}$. The second line runs horizontally, touching the line at its upper point with a value of $\beta_{\mathrm{w}}=-0.1^{\circ}$. The intersection of these lines forms a sector in which the organic deviation of relieved profile is practically absent.

\section{Profiling of grinding wheels}

Significant difficulties in the manufacture of worms and worm hobs with a profile of substantial and variable curvature are due to the fact that the circle dressing copiers included in the delivery of grinding machines are not suitable for reproducing such a profile $[1,2]$. At present, with the development of $\mathrm{CNC}$, ruling devices with diamond rollers and programmed control of their movement relative to the working surface of the grinding wheel have gained a certain distribution.

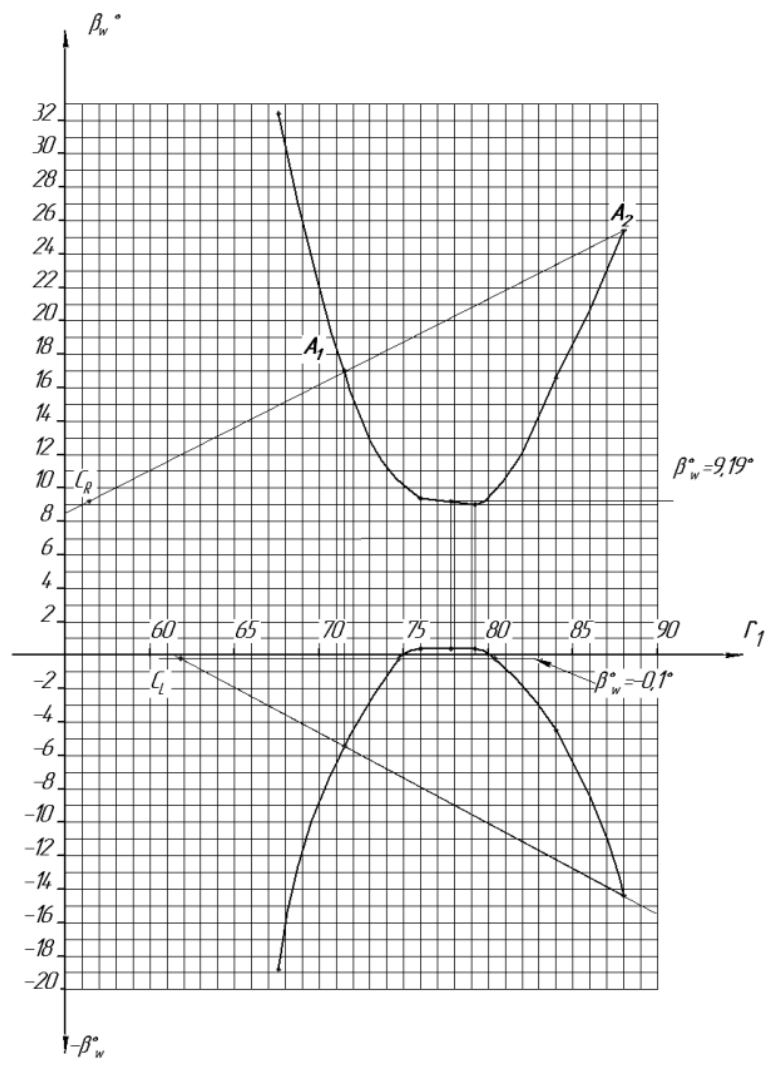

Fig. 6. Angles $\beta_{w}$ vs. the current radius of the hob.

The characteristic of the surface of the grinding wheel, i.e., the line of its contact with the surface to be ground, is oriented along the axial section of the latter. Therefore, profiling of the wheel in its axial section with a sufficient degree of approximation reproduces the section of the surface being ground that is normal to a helical line with the lead angle equal to $\beta_{\mathrm{w}}$. That is, the axial profile of the grinding wheel for single-thread hobs practically repeats the profile of the basic rack. In this case, the value of the angle $\alpha_{w}$ of the wheel profile in a rectilinear section is determined from the expression: 


$$
\tan \alpha_{\mathrm{w}}=\tan \left(\alpha_{0 \min }+\varphi_{0}\right) \cos \beta_{\mathrm{w}}
$$

In general, the coordinates of the axial profile of the grinding wheel, based on the coordinates of the axial section of the relieved surface of the hob teeth, the lead angles of this surface and the found angle of inclination of the wheel axis, are determined by the following expressions:

$$
\begin{gathered}
z_{\mathrm{w}}=z_{0 \mathrm{i}} /\left(\cos \beta_{\mathrm{w}}+\tan \gamma_{0 \mathrm{i}} \sin \beta_{\mathrm{w}}\right) \\
x_{\mathrm{w}}=x_{0 \mathrm{i}}+z_{\mathrm{n}}^{2} \sin 2 \beta_{\mathrm{w}} \tan \gamma_{0 \mathrm{i}} /\left(4 x_{0 \mathrm{i}}\right) .
\end{gathered}
$$

\section{Sensitivity to the hob regrinding}

If the relieving of surfaces with a variable curvature profile is performed by the radial-axial method, then the axial component $k_{\mathrm{x}}=k \sin \left(\alpha_{0 \mathrm{i}}+\varphi_{\mathrm{c}}\right) / \cos \alpha_{0 \mathrm{i}}$ of the relieving parameter included in formula (6) is variable along the profile. In this case, strictly speaking, the profile distortion during regrinding of the hob teeth on the front surface is inevitable. However, the considered profile has the points with identical pressure angles $\alpha_{0 \mathrm{i}}$ (for example, at the conjugate points of large and small radii on the tooth head and leg) and the lead angles $\gamma_{0 \mathrm{i}}$ of the relieved surface, and therefore, at these points and in the areas between them, the profile deviations as regrind are minimal.

\section{Conclusions}

The relieving process for tooth flanks of worm hobs for cutting Novikov gears with two lines of action has been studied. The results of this research are shown below.

1. Change of the relieved surface lead angle of hob teeth has a parabolic dependence on the radius and pressure angle of the tooth profile. The relieved tooth surfaces of such hobs are organically characterized by the presence of paired profile points with the equal lead angles.

2. The minimum rear angle of the hob teeth takes place near the hob pitch cylinder, and the installation angle $\varphi_{c}$ of the relieving support of the grindingrelieving machine is necessary to be calculated based on the minimum value of the rear angle $4^{\circ}$.

3. For both right and left sides of the teeth it is preferable to assign the installation angle $\varphi_{0}$ for the grinding wheel axis at $18^{\circ}$. Also, pruning on the opposite side of the generating surface of the cutting edges has to be prevented.

4. At the relieving of the left side of the right-hand hob teeth the directions of the lead angles of the relieving surface and the helical surface of the cutting edges have different signs. Accordingly, the angle $\beta_{\mathrm{w}}$ of inclination of the grinding wheel axis to the horizon can also have a sign opposite to the lead angle $\gamma_{1}$ of the hob generating surface.

The technique has been developed for calculating the parameters of installation, movement and profile of the disk grinding wheel for the relieving process of the tooth flanks. In the axial section of the hob it allows to obtain at least 4 points of contact between the wheel and the surface to be ground. Thus, the organic grinding error is minimized during the profiling of the grinding wheel, and generated profile is as close as possible to the profile of the hob basic rack.

The above technique is also proposed to be used in the development of control programs to set up the grinding-relieving machine and the dressing mechanism of the grinding wheel for worm hobs manufacturing on relieving machines with the numerical program control.

\section{References}

1. S.A. Lagutin and A.I. Sandler, Grinding of Helical and Relieved Surfaces, Publishing House "Mashinostroyeniye". (1991)

2. A.I. Sandler, S.A. Lagutin and A. V. Verhovski, Manufacturing of Worm Gears, Publishing House "Mashinostroyeniye" 272 p. (2008)

3. A.I. Sandler, S.A. Lagutin and E.A. Gudov, Theory and Practice of Manufacturing of General Type Worm Gears, Publishing House "Infra-Engineering" Moscow-Vologda (2016).

4. F.L. Litvin and A. Fuentes, Gear Geometry and Applied Theory, Cambridge University Press (2004)

5. A.I. Sandler and S.A. Lagutin, Features of Forming Screw and Relief Surfaces for Fluid Friction Worm Gears, International Symposium on Theory and Practice of Gearing, Izhevsk, pp. 375-380 (2014)

6. A.I. Sandler and S.A. Lagutin, Relieving the teeth of hobs for cutting the Wildhaber-Novikov gears. The 10th International Conference KOD 2018 "Machine and Industrial Design in Mechanical Engineering”, Novi Sad, Serbia / IOP Conference Series: Materials Science and Engineering, Volume 393, (2018)

7. M.L. Novikov, Gears with New Engagement, Publ. VVIA n.a. Zhukovsky (1958)

8. V. N. Kudryavtsev, On the issue of Novikov Gearing, In: Research and mastering of gears with Novikov gearing. Publ. House of the USSR Academy of Sciences. pp 33-44. (1960)

9. V.I. Korotkin, N.P. Onishkov and Yu. D. Kharitonov Novikov Gearing: Achievements and Development, Nova Science Publishers (2011)

10. $\mathrm{Yu}$. N. Stebletsov. A.S. Tarapanov, Wear resistance of the cutting tool when processing Novikov gears. In: Fundamental and applied problems of engineering and technology - Orel: State University - UNPK, - №4 / 3. Pp. 83-86 (2011) 